\title{
Six Transmembrane Epithelial Antigen of Prostate (STEAP1) Immunohistochemical Expression in Neoplastic and Non-Neoplastic Epithelial Lesion of Prostate
}

\author{
Fatma M.K.H. Ibrahim*, Elsayed A. Ali, Maha M.F. Shamloula, Mohamed M. Shareef \\ and Asmaa E. Bedeer
}

Pathology Department, Faculty of Medicine, Tanta University, Egypt

*Corresponding author

K e y w o r d s
Prostatic
adenocarcinoma, HGPIN,
STEAP1,
Immunohistochemistry,
Mimickers of prostatic
carcinoma

\section{Introduction}

Prostatic carcinoma (PCa) is one of the leading causes of morbidity and mortality in the developed countries and the incidence of prostatic carcinoma is rapidly rising in developing countries due to significant westernization among these populations (Djavan et al., 2011). The currently used biomarkers including prostatic specific antigen
Prostatic carcinoma is one of the most common malignancies in the world. Considering the incidence and the mortality of prostate cancer it seems important to study novel biomarkers for prostate cancer diagnosis and progression. Study the immunohistochemical expression of STEAP1 in prostatic adenocarcinoma, HGPIN and benign epithelial lesions of prostate stressing on its role in distinguishing prostatic carcinoma from its benign mimickers. STEAP1 expression was evaluated immunohistochemically in 30 cases of benign prostatic lesions, 8 cases of HGPIN and 40 cases of prostatic adenocarcinoma, and the results was correlated with clinicopathological and histopathological features. Significant difference was detected between STEAP1 expression in benign and malignant prostatic lesions, including prostatic adenocarcinoma and HGPIN, but no significant difference was detected between STEAP1 expression in prostatic carcinoma and HGPIN cases. Moreover, significant relationship was detected between STEAP1 expression and Gleason score of the adenocarcinoma cases. STEAP1 could be considered as an immunohistochemical marker with good sensitivity and considerable specificity for differentiating between benign and malignant prostatic lesions (HGPIN and prostatic adenocarcinoma) but it lacks specificity in distinguishing prostatic adenocarcinoma from HGPIN. STEAP1 might be considered as prognostic marker for prostatic adenocarcinoma and its overexpression was more associated with higher Gleason score.

A B S T R A C T

(PSA and prostatic specific acid phosphatase (PSAP) don't provide the adequate sensitivity and specificity for prostatic cancer diagnosis. To this end, it becomes necessary to study novel biomarkers for the accurate diagnosis of prostatic cancer at an early stage, so that patients can get the benefit of early therapeutic intervention (Bhavsar et al., 2013). Although the diagnosis of prostatic carcinoma can usually be made on morphological basis 
including infiltrative growth pattern, nuclear atypia and the absence of basal cells, it is sometimes difficult to reach firm diagnosis by routine histopathological evaluation, in particular for small foci of carcinoma in needle biopsy. This is not only because there is no single specific diagnostic feature but also because many benign conditions can mimic prostatic carcinoma. Therefore, the application of immunohistochemistry to distinguish prostate carcinoma from its mimickers and to confirm the diagnosis would be helpful and necessary especially for equivocal cases (Verma and Jasani, 2005).

Alpha methyl coenzyme A racemase (AMACR) (P504), an enzyme involved in lipid metabolism, is a frequently used immunohistochemical marker to distinguish prostatic adenocarcinoma from benign glandular proliferation. AMACR is upregulated in malignant cells including invasive adenocarcinoma and HGPIN, whereas benign glands generally lack this marker (Carswell et al., 2006). However, AMACR immunostaining cannot be used as the sole argument for a definite diagnosis of malignancy. About $20 \%$ of benign prostatic glands, including atrophy and adenosis, may express AMACR, but the staining is usually more focal and weaker than carcinoma.

Additionally, about 20\% of limited adenocarcinoma show negative staining for AMACR. It was also demonstrated that pseudohyperplastic and atrophic variants of prostatic adenocarcinoma, that are particularly difficult to diagnose, are less frequently (62$77 \%$ ) positive for AMACR. Moreover, AMACR is not entirely specific for prostatic carcinoma and its overexpression has been reported in many other tumors, such as colorectal, ovarian, breast, bladder, lung, and papillary renal cell carcinomas, as well as in lymphoma and melanoma (Herawi and Epstein, 2007).
Six transmembrane epithelial antigen of the prostate 1 (STEAP1) is a transmembrane protein of epithelial cells, mostly located at cell-cell junctions, possibly involved in transmembrane electron transfer (Gomes et al., 2014). STEAP1 is virtually not expressed in normal tissues. In contrast, it is strongly overexpressed in many carcinomas including prostate, breast, and bladder carcinoma (Maia et al., 2008).

Grunewald et al., have also demonstrated STEAP1 as membrane bound mesenchymal stem cell marker protein that is highly expressed in sarcomas such as Ewing Sarcoma. In addition, it was recently showed that STEAP1 overexpression increases the invasive properties and intracellular levels of reactive oxygen species (ROS) of Ewing Sarcoma cells (Grunewald et al., 2012). Moreover, the successful generation of two monoclonal antibodies $(\mathrm{mAb})$ that bind to cell surface STEAP1 epitopes has provided the tools to study STEAP1 susceptibility to naked antibody therapy. Studies have also shown STEAP-1 as a suitable antigen for T-cellbased immunotherapy; however, its utilization for antibody-mediated targeting has not yet been explored (Challita-Eid et al., 2007).

\section{Materials and Methods}

This retrospective study was carried out on 78 paraffin blocks of prostatic lesions, which were divided into 3 groups, collected from archive of Pathology department, Faculty of Medicine, during the period from April 2015 to May 2017 including:

30 cases of benign prostatic epithelial lesions 40 cases of prostatic adenocarcinoma 8 cases of HGPIN

Clinical data such as age, total serum PSA level and type of biopsy specimen were obtained from clinical sheets. 
Approval from research ethics committee (REC), Faculty of Medicine, was taken prior to conducting the study

\section{Histopathological study}

Serial sections were prepared from each block. Histological sections, 4-mm thick, were stained by hematoxylin and eosin $(H \& E)$ for evaluation of histopathological parameters.

Prostatic adenocarcinoma cases were classified according to 2016WHO classification of prostatic tumors.

Adenocarcinoma cases were graded according to the modified Gleason scoring system.

\section{Immunostaining}

From each paraffin block, 4-mm-thick sections mounted onto positively charged slides were taken for immunohistochemical staining using STEAP1 primary antibody (Clone H-105, Cat. No. NS1756-S0, concentrated, Santa Cruz biotechnology, USA).

The secondary antibody used was Biotinylated Goat Anti-Mouse IgG (H+L) (Lab vision, USA, Cat No. TM-060-BN), diluted, ready to use.

\section{Assessment of immunostaining}

STEAP1 immunoreactivity was assessed semiquantitatively using a grade score system, based on the intensity of staining and percentage of prostatic luminal cells as following:

\section{The staining intensity was classified as}

"0": (no staining)

"1": (weak staining)

"2": (high staining)
The percentage of stained cells were classified as

" 0 ": (no stained cells)

"1": (up to $25 \%$ of stained cells)

" 2 ": (between $25 \%$ and $50 \%$ of stained cells)

" 3 ": (more than $50 \%$ of stained cells)

Subsequently, a final score was obtained by multiplying the percentage of stained luminal cells and intensity of staining. Then, these score values were grouped into:

"Low score": (scores 0 and 1)

"Moderate score": (scores 2and3)

"High score": (scores 4and6).

\section{Statistical analysis}

Database and data analyses were done with IBM SPSS Statistics 20. Descriptive and analytical methods were used. For comparison of variables, parametric ( $\mathrm{t}$ test, ANOVA) and non-parametric tests (chi square were employed. With objective to compare markers Receiver Operating Curve (ROC) was constructed. Sensitivity, specificity, positive and negative predictive values were calculated for single and combination of markers in program MedCalc $®$ (Version 10.2.0.0). Probability values less than 0.05 were considered statistically significant

\section{Results and Discussion}

Histopathological results of the studied cases

The present work included 78 cases of prostatic lesions consisting of 30 cases of benign prostatic lesions, 8 cases of HGPIN and 40 cases of prostatic adenocarcinoma.

Out of the studied 30 cases of benign prostatic lesions, 12 cases were pure benign prostatic hyperplasia (BPH) and the remaining 18 cases 
were of benign mimickers of prostatic adenocarcinoma including 8 cases of prostatic atrophy, 8 cases of adenosis (Fig. 1), 2 cases of reactive glandular atypia and 3 cases of basal cell hyperplasia (Fig. 2).

The 8 cases of HGPIN included 3 cases with flat pattern, 3 cases with tufting pattern and two cases with micropapillary pattern (Fig. 3). The 40 cases of prostatic adenocarcinoma included 37 cases of acinar adenocarcinoma and its variants and 3 cases of ductal adenocarcinoma (Fig. 4). Eleven cases were of Gleason scores $5 \& 6$ while the remaining 29 cases were of Gleason score 7 or more, of which 7 cases were of Gleason score 7,11 cases of score 8 (Fig. 5), 5 cases of score 9 and 6 cases of score 10 .

\section{Immunohistochemical results}

STEAP1 expression in benign prostatic lesions

Moderate score cytoplasmic and/or membranous expression of STEAP1 was detected in 7 out of 30 benign cases, including 3 cases of BPH, 1 case of atrophy, 2 cases of adenosis and one case of reactive atypia, while the remaining 23 cases showed low to negative staining (score $0 \& 1$ ).

\section{STEAP1 expression in HGPIN cases}

Regarding HGPIN cases, high score of STEAP1 expression was detected in 6 out of the studied 8 cases (Fig. 6), while moderate score expression was detected in the remaining 2 cases.

\section{STEAP1 expression in prostatic adenocarcinoma cases}

Moderate score of STEAP1 expression was detected in 14 cases out of studied 40 cases of prostatic adenocarcinoma (Fig. 7); while 23 cases showed high score (Fig. 8 and 9) and the remaining 3 cases showed low to negative (score $0 \& 1$ ) of expression.

STEAP1 expression in benign and malignant lesions (Table 1\&2)

Significant difference was detected between STEAP1 expression in benign and malignant prostatic lesions, including prostatic adenocarcinoma and HGPIN supporting its role in distinguishing benign from malignant prostatic lesions. However, no significant difference was detected between STEAP1 expression among prostatic adenocarcinoma and HGPIN cases.

Relationship between STEAP1 expression and Gleason scoring of adenocarcinoma cases (Table 3)

STEAP1 expression was significantly correlated with the Gleason score of the prostatic adenocarcinoma cases.

\section{Sensitivity and specificity of STEAP1 (Table 4)}

STEAP1 immunoreactivity showed good discriminatory power in distinguishing between benign and malignant prostatic lesions (adenocarcinoma and HGPIN), with sensitivity and specificity of $94 \%$ and $74 \%$ respectively. However the sensitivity of STEAP1 to detect prostatic adenocarcinoma and not HGPIN is lowered to $57 \%$ with $25 \%$ specificity.

Prostate cancer is the sixth most common cancer in the world, and together with lung and colon cancer, it is the most frequent form of malignancy among men (Jemal et al., 2007). Prostatic carcinoma is a clinically, morphologically and molecularly heterogeneous disease (Ihlaseh-Catalano et al., 2013). 
Table.1 STEAP1 expression in all the studied cases

\begin{tabular}{|c|c|c|c|c|}
\hline $\begin{array}{c}\text { Histopathological } \\
\text { diagnosis }\end{array}$ & $\begin{array}{l}\text { Low } \\
\text { score } \\
(0,1)\end{array}$ & $\begin{array}{c}\text { Moderate } \\
\text { score } \\
(2,3)\end{array}$ & $\begin{array}{c}\text { High } \\
\text { score } \\
(4,6)\end{array}$ & total \\
\hline $\begin{array}{l}\text { Benign prostatic } \\
\text { lesions }\end{array}$ & $23(76.7)$ & 7 (23.3) & 0 & $\begin{array}{c}30 \\
(100 \\
\% 0\end{array}$ \\
\hline HGPIN & 0 & $2(25 \%)$ & $6(75 \%)$ & 8 \\
\hline $\begin{array}{c}\text { prostatic } \\
\text { adenocarcinoma }\end{array}$ & $3(7.5 \%)$ & $14(35 \%)$ & $\begin{array}{c}23(57.5 \% \\
)\end{array}$ & $\begin{array}{c}40 \\
(100)\end{array}$ \\
\hline P1 & \multicolumn{4}{|c|}{$<0.001 *$} \\
\hline $\mathbf{P 2}$ & \multicolumn{4}{|c|}{0.5} \\
\hline
\end{tabular}

P1: * Highly significant difference between STEAP1 expression in benign prostatic lesions and HGPIN ( $\mathrm{p}$ value less than 0.05 ).

P2: No significant difference was detected between STEAP1 expression in HGPIN \& prostatic adenocarcinoma cases (P value more than 0.05 ).

Table.2 STEAP1 expression in benign and malignant prostatic lesions

\begin{tabular}{|c|c|c|c|c|}
$\begin{array}{c}\text { Histopathological } \\
\text { diagnosis }\end{array}$ & $\begin{array}{c}\text { Low score } \\
(\mathbf{0}, \mathbf{1})\end{array}$ & $\begin{array}{c}\text { Moderate } \\
\text { score }(\mathbf{2}, 3)\end{array}$ & $\begin{array}{c}\text { High } \\
\text { score } \\
(4,6)\end{array}$ & total \\
\hline $\begin{array}{c}\text { Benign prostatic } \\
\text { lesions }\end{array}$ & $\begin{array}{c}23 \\
(76.7 \%)\end{array}$ & $7(23.3 \%)$ & $0(0 \%)$ & $\begin{array}{c}30(100 \\
\%)\end{array}$ \\
\hline $\begin{array}{c}\text { Malignant } \\
\text { lesions (HGPIN } \\
\text { \& PCa) }\end{array}$ & $3(6.2 \%)$ & $16(33.3 \%)$ & $\begin{array}{c}29 \\
(60.4 \%)\end{array}$ & $\begin{array}{c}48 \\
(100 \%)\end{array}$ \\
\hline X2 & & $\mathbf{4 6 . 2}$ & \\
\hline P value & $\mathbf{3 0 . 0 0 1 *}$ & \\
\hline
\end{tabular}

Table.3 Relation between STEAP1 immmuno expresssion and Gleason scoring of the studied prostatic adenocarcinoma cases

\begin{tabular}{|c|c|c|c|c|}
\hline \multirow[t]{2}{*}{ Gleason score } & \multicolumn{3}{|c|}{$\begin{array}{l}\text { Scoring of STEAP1 expression in prostatic } \\
\text { adenocarcinoma }\end{array}$} & \multirow[t]{2}{*}{ total } \\
\hline & Low score & Moderate score & high score & \\
\hline Gleason score $<7$ & $\mathbf{0}(\mathbf{0} \%)$ & $8(72.7 \%)$ & $3(7.2 \%)$ & $11(100 \%)$ \\
\hline $\begin{array}{c}\text { Gleason score } \overline{ } \\
7 \\
\end{array}$ & $3(10.3 \%)$ & $6(20.6 \%)$ & $20(69 \%)$ & $29(100 \%)$ \\
\hline $\mathrm{X} 2$ & \multicolumn{4}{|c|}{9.71} \\
\hline P-value & \multicolumn{4}{|c|}{$0.007 *$} \\
\hline
\end{tabular}


Table.4 Sensitivity and specificity of STEAP1

\begin{tabular}{|l|c|c|c|c|c|c|}
\hline Diagnosis & Cut off & sensitivity & Specificity & $\begin{array}{c}\text { Positive } \\
\text { predictive } \\
\text { value }\end{array}$ & $\begin{array}{c}\text { Negative } \\
\text { predictive } \\
\text { value }\end{array}$ & Accuracy \\
\hline $\begin{array}{l}\text { Malignant vs. } \\
\text { Benign lesions }\end{array}$ & $\begin{array}{c}\text { Low } \\
\text { (score1) }\end{array}$ & $\mathbf{9 4 \%}$ & $\mathbf{7 4 \%}$ & $\mathbf{8 6 . 5 \%}$ & $\mathbf{8 8 \%}$ & $\mathbf{8 7 \%}$ \\
\hline $\begin{array}{c}\text { Adenocarcino } \\
\text { ma Vs HGPIN }\end{array}$ & $\begin{array}{c}\text { Moderate } \\
\text { (score3) }\end{array}$ & $\mathbf{5 7 \%}$ & $\mathbf{2 5 \%}$ & $\mathbf{7 9 \%}$ & $\mathbf{1 2 . 5 \%}$ & $\mathbf{5 2 \%}$ \\
\hline
\end{tabular}

The cutoff in distinguishing malignant from benign lesions means that cases with moderate or high STEAP1 immunoreactivity (scores more than1) would be considered as having malignant lesion.

The cutoff in distinguishing prostatic adenocarcinoma from HGPIN means that individuals with low or moderate STEAP1 immunoreactivity (scores less than 3 ) would be considered as having HGPIN.

Fig.1 A case of prostatic adenosis (atypical adenomatous hyperplasia) showing nodule of small crowded glands merging with larger ones (H\&E x200)

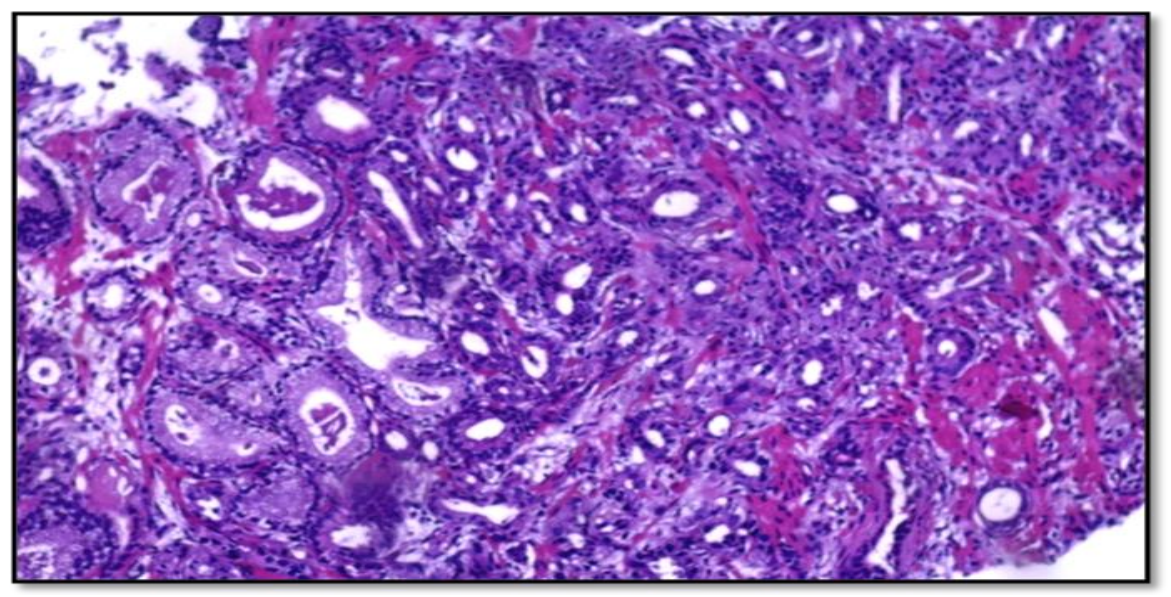

Fig.2 A case of basal cell hyperplasia showing multiple glands in which luminal circumference is surrounded by multiple layers of basal cells (H\&E x200)

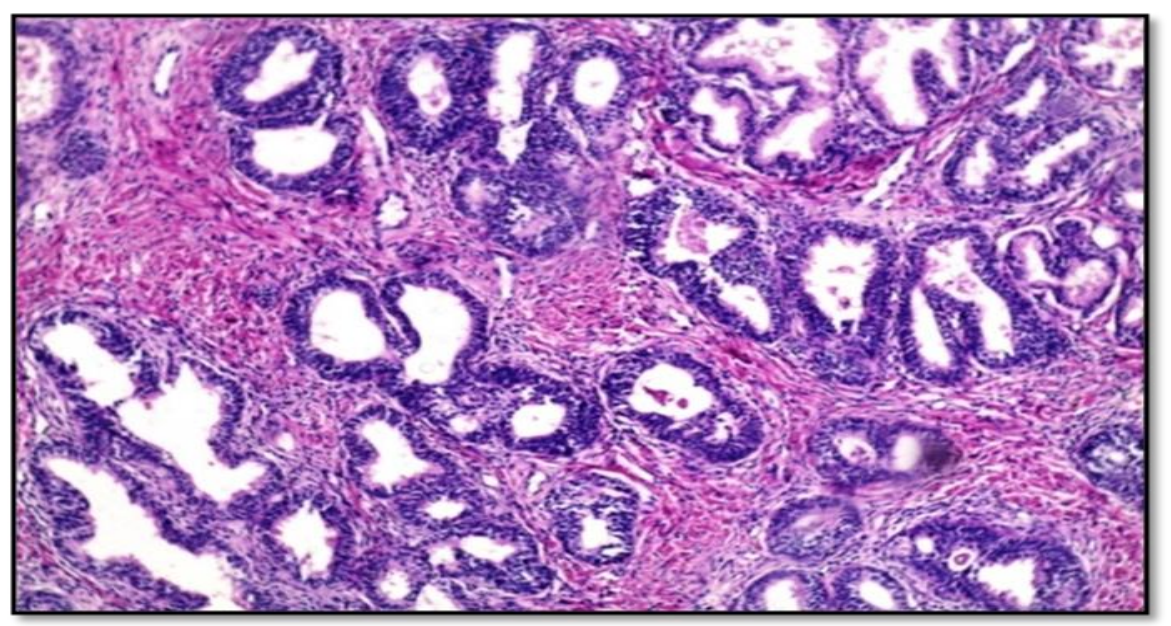


Fig.3 A case of HGPIN (micropapillary pattern) showing intraluminal elongated tufts and columns of cells without fibrovascular core incompletely bridging the acinar lumina (H\&E x400)

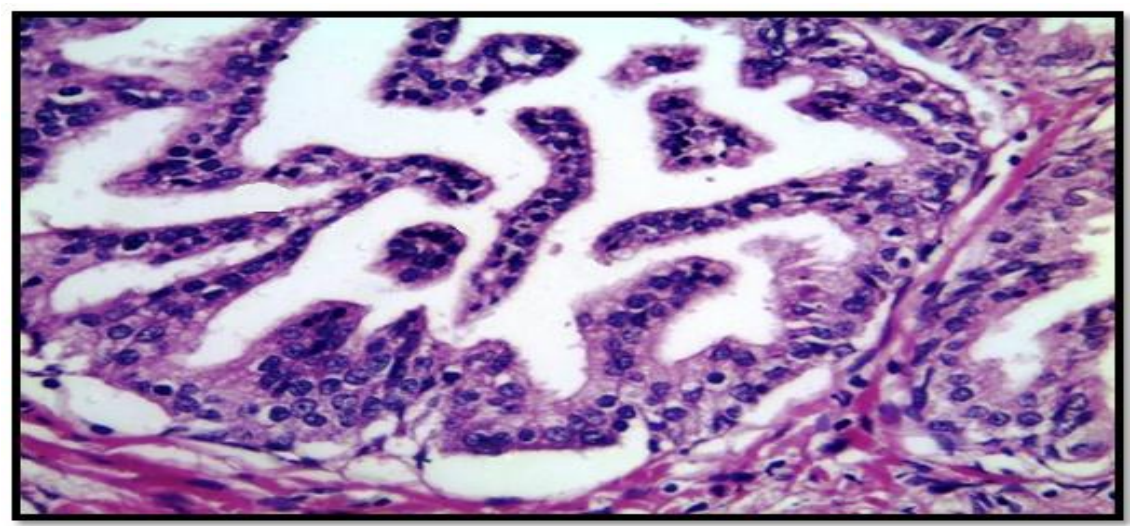

Fig.4 A case of prostatatic ductal adenocarcinoma Gleason grade 5 (a component of score 9) showing cribriform glands and papillary formation with central comedo necrosis (H\&E x400)

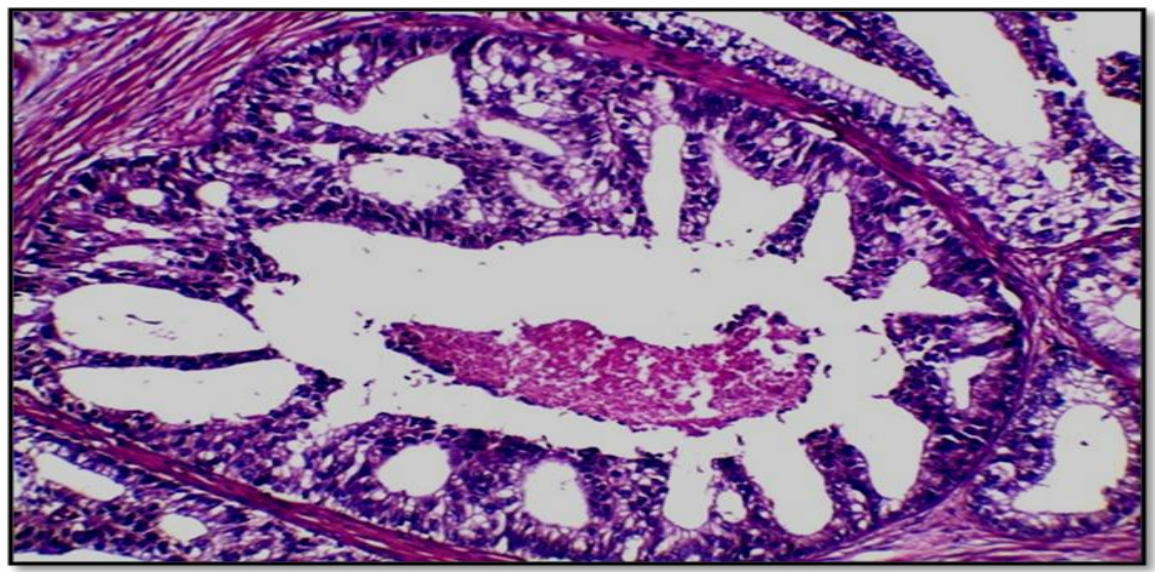

Fig.5 A case of prostatic adenocarcinoma, Gleason grade 4 (a component of score 8) showing fused malignant glands in cribriform pattern lined by cells with enlarged nuclei and prominent nucleoli (H\&E x400)

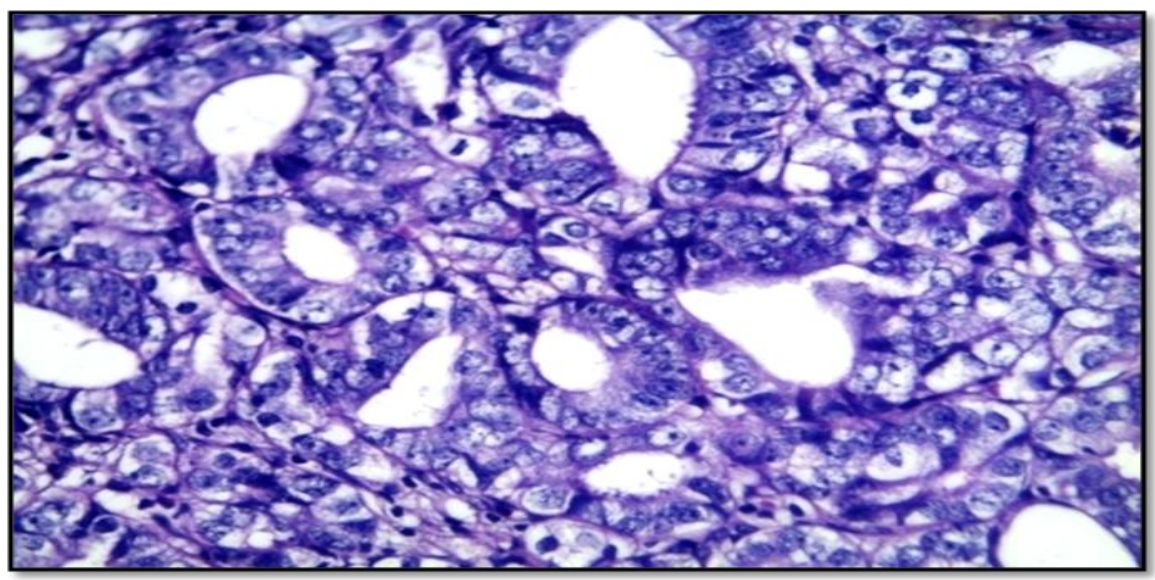


Fig.6 A case of HGPIN (tufting pattern) showing high score (6) [intensity 2X percentage 3] cytoplasmic expression of STEAP1 in the luminal cells of involved glands

(Immunohistochemistry X 400)

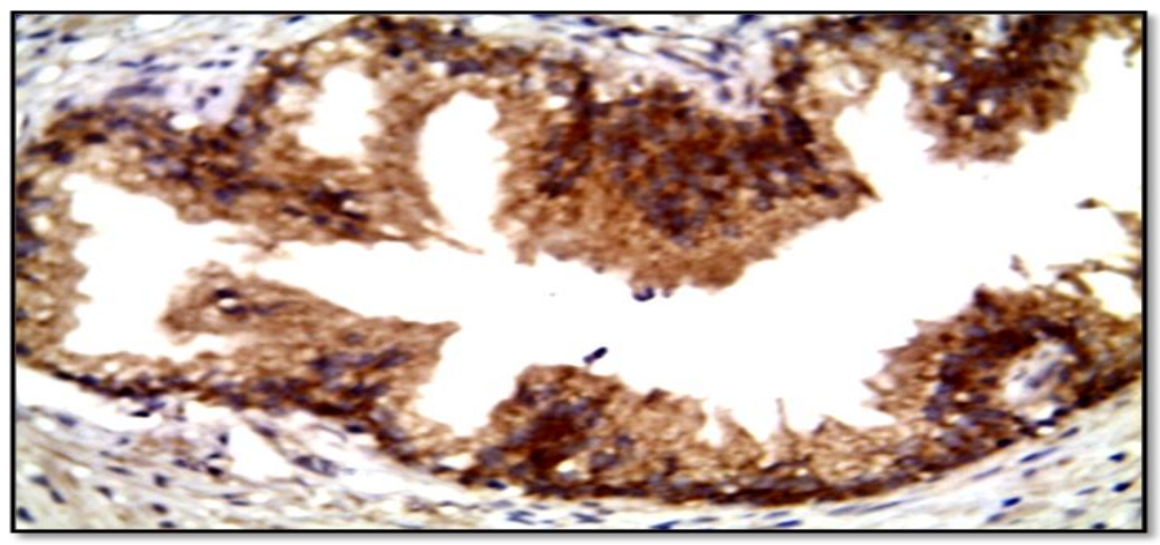

Fig.7 A case of prostatic adenocarcinoma Gleason grade 3 (a component of Gleason score 6) showing moderate score (3) [intensity $1 \mathrm{X}$ percentage 3] cytoplasmic expression for STEAP1 in the neoplastic glands (Immunohistochemistry X 400)

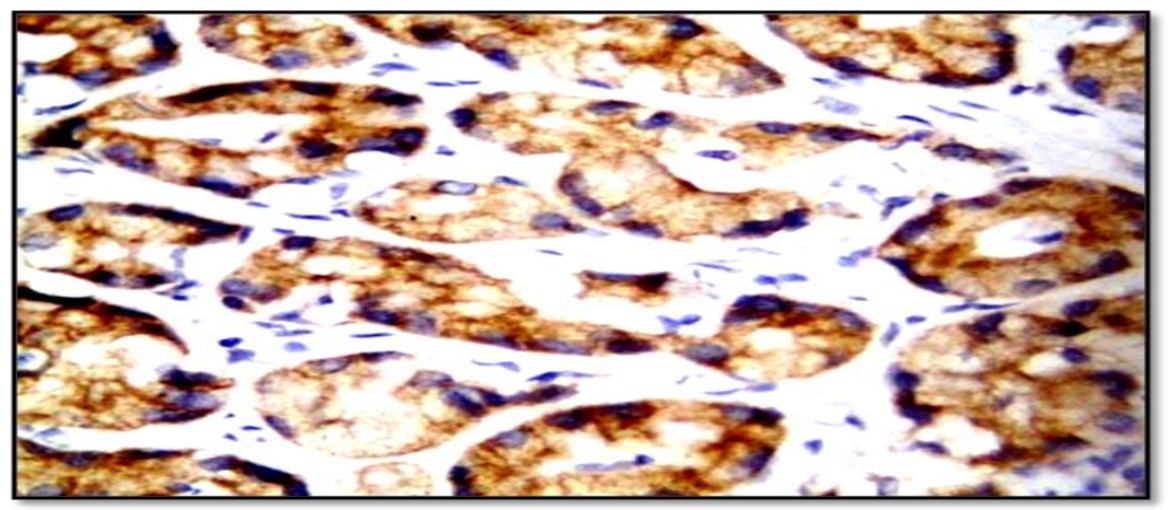

Fig.8 A case of prostatic adenocarcinoma, Gleason grade 4 (a component of Gleason score 8) showing high score (6) [intensity $2 \mathrm{X}$ percentage 3 ] cytoplasmic expression of STEAP1 in the neoplastic cells (Immunohistochemistry X 200)

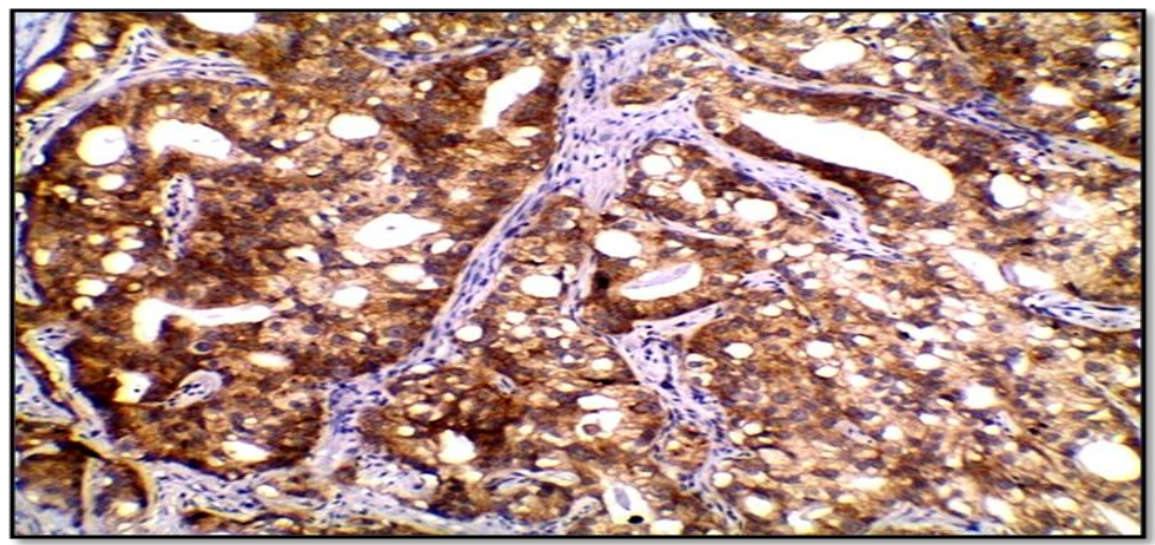


Fig.9 A case of prostatic adenocarcinoma, Gleason grade 4 (a component of Gleason score 8) showing high score (6) [intensity $2 \mathrm{X}$ percentage 3 ] cytoplasmic expression of STEAP1 in the neoplastic cells (Immunohistochemistry X 400)

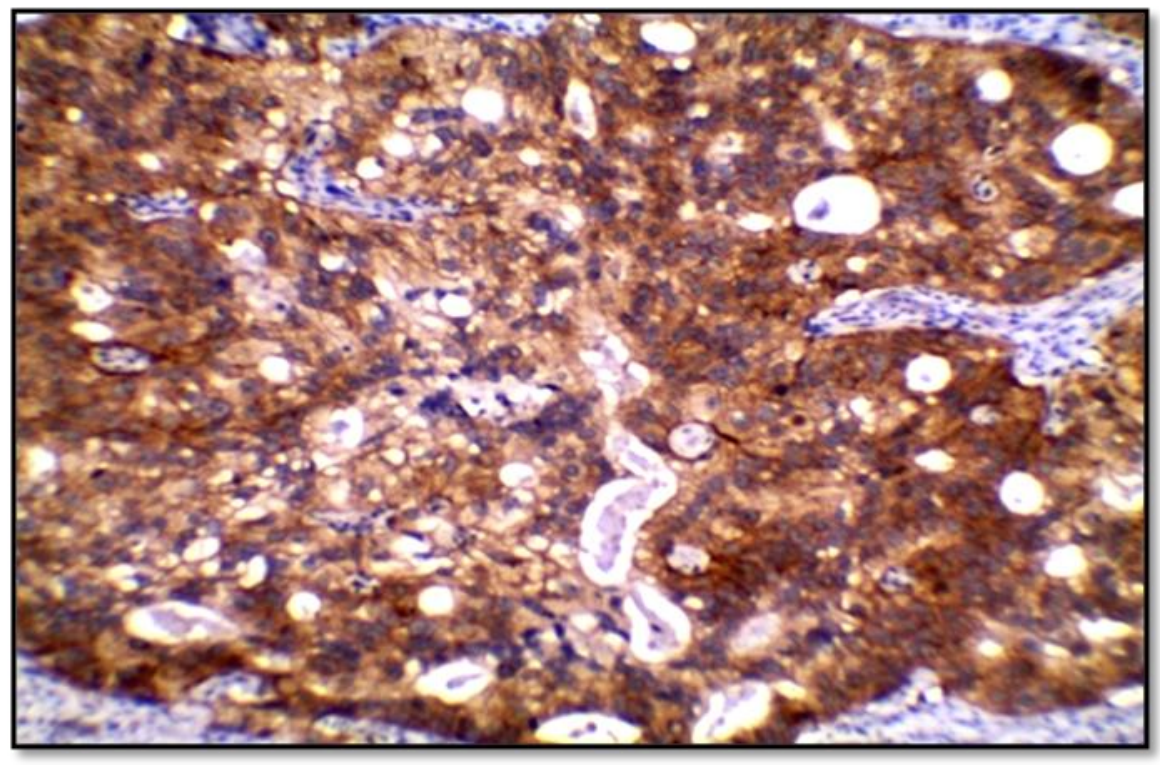

Considering the incidence and the mortality of prostate cancer and the limitations of the serum PSA level as a marker for this disease, it seems important to study novel putative diagnostic and prognostic biomarkers for prostatic cancer (Gomes et al., 2013).

STEAP1 is primarily localized at the plasma membrane, mostly at cell-cell junctions, but it may also be found dispersed in the cytoplasm (Maia et al., 2008). The cytoplasmic expression of STEAP1 may suggest that it can be linked to cytoplasmic organelles. Given its localization on the cell membrane and its predicted secondary structure, as a 6transmembrane protein, STEAP1 is expected to act as a membrane channel or transporter protein in tight and gap junctions taking part in intercellular communication. Since STEAP1 is overexpressed in several types of cancer, it has been suggested that STEAP1 may facilitate cancer cell proliferation and invasion, perhaps through regulation of ion concentration such as $\mathrm{Na}, \mathrm{K}$, and $\mathrm{Ca} 2$ (Chalita-Eid et al., 2007 and Grunewald et al., 2012). STEAP1 has restricted expression in normal tissues, except for bladder tissue which exhibit little expression (Hubert et al., 1999), but a wide expression in several types of cancers, including breast, cervix, pancreas, bladder, colon, ovary, testis and Ewing sarcoma (Yang et al., 2001 and Mai et al., 2008). The strategic localization of STEAP1 at the cell surface, its low expression in normal tissues and its overexpression in many cancerous tissues mark this protein as a potential target for diagnosis and therapy of cancer.

In the current work, 7 cases $(23.3 \%)$ out of 30 cases with benign prostatic lesions showed moderate score cytoplasmic and or membranous immunoreactivity for STEAP1, including 3 cases of BPH, 1 case of atrophy, 2 cases of adenosis and one case of reactive atypia. The remaining 23 cases showed negative to low STEAP1 expression. These results were different from the results of the study carried out by Gomes et al., 2014 where only 2 out of 41 cases of BPH $(4.88 \%)$ showed moderate score cytoplasmic and membranous expression of STEAP1. This may be due to 
lack of inclusion of benign cancer mimickers in their study. In a study by Ihlaseh- Catalano et al., 2013, they detected weak membranous staining of STEAP1 in BPH and normal prostatic tissue samples. However, they adopted different system for interpretation of STEAP1 expression depending on intensity of staining only.

All the studied cases of HGPIN included in the current study showed STEAP1 immunoreactivity with different scoring of expression. Moderate score of STEAP1 expression was detected in $2 / 8(25 \%)$ of cases, while the remaining 6 cases $(75 \%)$ showed high score of expression. These results were close to those of Gomes et al., (2014) who detected moderate expression among $1 / 7 \quad(14.2 \%)$ and high expression among 6/7 (85.7\%) of HGPIN cases. HGPIN is considered as premalignant lesion in prostate. The expression of STEAP1 in HGPIN lesions may suggest that STEAP1 is overexpressed even before prostate cancer development and that it has an important role in prostate cancer initiation and progression.

In the current work, moderate score of STEAP1 expression was observed in 14 cases (35\%) out of studied 40 cases of prostatic adenocarcinoma; while 23 cases $(57.5 \%)$ showed high score and the remaining 3 cases (7.5\%) showed low to negative score of expression. Our results were in agreement with Gomes et al., (2014) who found that $36.5 \%$ of prostatic carcinoma cases showed moderate score of STEAP1 expression, while $52.3 \%$ showed high score and $11.1 \%$ showed negative to low score of expression. The three cases of prostatic adenocarcinoma with low score (0\&1) STEAP1 expression, were 2 negatively stained cases of ductal adenocarcinoma (Gleason scores 8\&9) and one case of foamy gland carcinoma (low score 1), suggesting the need for further studies to evaluate STEAP1 expression among different variants of prostatic ductal and acinar adenocarcinoma.

In the current work, a significant difference was detected between STEAP1 expression in benign and malignant prostatic lesions, including prostatic adenocarcinoma and HGPIN, ( $p$ value less than 0.001), supporting its role in distinguishing benign from malignant prostatic lesions. However, no significant difference was detected between STEAP1 expression in prostatic adenocarcinoma and HGPIN cases (P value more than 0.05). Similar results were obtained by Gomes et al., (2014). Moreover, IhlasehCatalano et al., (2013) detected weak membranous expression among cases of BPH \& normal prostatic tissue and moderate to strong membranous and cytoplasmic staining among $82 \%$ of prostatic carcinoma cases. Their results were statistically significant. They also found that STEAP1 m-RNA transcript level was higher among cases of prostatic carcinoma than BPH and adjacent non neoplastic tissue, although the results were not statistically significant. Gomes et al., 2012 detected little or no expression of STEAP1 m-RNA transcript on the nonneoplastic prostate cell lines and high expression on the malignant prostate cell lines. They hypothesized that STEAP1 mRNA is more stable in prostatic cancer cell lines than in non-neoplastic cell lines. On the contrary, Hubert et al., 1999 demonstrated moderate to strong STEAP1 immunohistochemical staining in glandular epithelia of all prostate cancer samples and in all samples derived from normal prostate or benign prostatic lesions. These discordant results can, in part, be explained by the different antibodies used to detect STEAP1 and the small number of tissue samples (only 26) evaluated by these authors.

In our work, a highly significant relationship was detected between STEAP1 expression 
and Gleason score of the studied adenocarcinoma cases. High percentage $(68.9 \%)$ of patients with higher Gleason score (7-10) displayed high score STEAP1 immunoreactivity, whereas in cases with Gleason scores (5-6) 27.2\% of cases exhibited high score STEAP1 immunoreactivity and $72.7 \%$ of cases showed moderate score of expression. Gomes et al., 2014 have also detected significant association between STEAP1 overexpression and various prognostic parameters of prostatic adenocarcinoma including Gleason score. These results suggest the role of STEAP1 as prognostic marker for prostatic adenocarcinoma. Furthermore, IhlasehCatalano et al., 2013 detected significant association between STEAP1 overexpression and biochemical recurrence of prostatic carcinoma (postsurgical serum PSA levels $\geq$ $0.2 \mathrm{ng} / \mathrm{ml})$. The same author's detected STEAP1 protein overexpression (by immunohistochemistry) and higher level of STEAP1 m-RNA transcript (by PCR) among prostatic adenocarcinoma cases with higher Gleason score and higher stage, although the results weren't statistically significant. The previous results were in accordance with Grunewald et al., (2012) who detected that STEAP1 overexpression is associated with aggressive phenotype, yet with better treatment outcome in Ewing sarcoma. Moreover, Moeureux et al., (2012) detected that STEAP1 overexpression in colorectal carcinoma, myeloid leukemia and B cell lymphoma was associated with poor survival rates.

It is suggested that an androgen independent stage develop with advanced Gleason score and may be associated with poor prognosis. Gomes et al., (2013) noticed that Dihydrotetosterone (DHT) markedly decreased the expression of STEAP1 in prostate cancer cell line. In vivo results demonstrated that castration increases
STEAP1 protein expression when compared to intact rats, and treatment with DHT reversed the effect of castration on STEAP1 expression, suggesting that STEAP1 protein is down-regulated by DHT. Since STEAP1 is down-regulated by androgens and STEAP1 may facilitate cell proliferation, it is expected that androgen refractory tumors with higher levels of STEAP1 may be more aggressive than tumors with lower levels of STEAP1. On the contrary, Hubert et al., (1999) demonstrated no difference between STEAP1 expression among tissue samples of all stages of prostate cancer, including low grades, high grades, and metastatic prostate cancer specimens. The same authors noticed that in prostate cancer cell lines, STEAP1 expression is increased in early prostate cancer stages in opposition to advanced stages.

In fact the development of markers and therapeutic targets for advanced androgen independent stage of prostate cancer is essential for improving diagnosis and treatment of prostatic carcinoma. Ideal tumor marker and therapeutic target should have restricted normal indispensible tissue expression but expressed well in advanced metastatic disease and should be accessible to therapy (cell surface expression) (Hubert et al., 1999).

Low number of radical prostatectomy specimens and the lack of some clinicopathological data among the included cases of prostatic adenocarcinoma in the current study didn't allow studying of the association between STEAP1 expression and other prognostic parameters of prostatic adenocarcinoma such as staging. Limited number of ductal adenocarcinoma and variants of acinar adenocarcinoma was another limitation of the current work that interfered with evaluation of the role of STEAP1in diagnosis of different variants of prostatic adenocarcinoma. 
STEAP1 could be considered as an immunohistochemical marker with good sensitivity and considerable specificity for distinguishing benign from malignant prostatic lesions (HGPIN \& prostatic adenocarcinoma), however, it lacks sensitivity and specificity for distinguishing prostatic adenocarcinoma from HGPIN. More studied are still in need to evaluate STEAP1 expression in uncommon variants of prostatic acinar and ductal carcinoma. STEAP1 may be also considered as prognostic marker for prostatic adenocarcinoma and its overexpression was more associated with higher Gleason score.

\section{References}

Bhavsar T., McCue P., and Birbe R. (2013): Diagnosis of Prostate Cancer: Are We Upto Age? Semin Oncol; 40:259-275.

Carswell BM, Woda BA, Wang X et al., (2006): Detection of prostate cancer by alpha-methylacyl Co A racemase $(\mathrm{P} 504 \mathrm{~S})$ in needle biopsy specimens previously reported as negative for malignancy. Histopathology; 48; 668673.

Challita-Eid M. P., Morrison K, Etessami S. et al., (2007): Monoclonal Antibodies to Six Transmembrane Epithelial Antigen of the Prostate-1 Inhibit Intercellular Communication In vitro and Growth of Human Tumor Xenografts In vivo. Cancer Research; 67: 5798-805

DjavanB, Kazzazi A, Dulabon L et al., (2011): Diagnostic Strategies for Prostate Cancer. European urology suplement; 10:26-37.

Gomes IM, Maia CJ and Santos CR. (2012): STEAP proteins: from structure to applications in cancer therapy. Mol Cancer Res; 10:573-87.

Gomes IM, Santos CR, Socorro S and Maia CJ (2013): Six transmembrane epithelial antigen of the prostate 1 is down regulated by sex hormones in prostate cells. Prostate; 73: 605-13.

Gomes MI, Arinto P, Lopes C et al., (2014): STEAP1 is overexpressed in prostate cancer and prostatic intraepithelial neoplasia lesions, and it is positively associated with Gleason score. Urologic Oncology: Seminars and Original Investigations; 32 53.e23-53.e29.

Grunewald P.G.T., Ranft A., Esposito I. et al., (2012): High STEAP1 expression is associated with improved outcome of Ewing's sarcoma patients. Ann Oncol; 23: 2185-90.

Herawi $M$ and Epstein JI (2007): Immunohistochemical antibody cocktail staining (p63/HMWCK/AMACR) of ductal adenocarcinoma and Gleason pattern 4 cribriform and noncribriform acinar adenocarcinomas of the prostate. Am J Surg Pathol; 31:889-894.

Hubert RS, Vivanco I, Chen E et al., (1999): STEAP: a prostate specific cell- surface antigen highly expressed in human prostate tumors. Proc Natl Acad Sci USA; 96: 14523-8.

Ihlaseh-Catalano MS, Drigo, SA, de Jesus MNC et al., (2013): STEAP1 protein overexpression is an independent marker for biochemical recurrence in prostate carcinoma. Histopathology, 63: 678-685.

Jemal A, Siegel R, Ward E et al., (2007): Cancer statistics, CA Cancer $\mathrm{J}$ Clin 57:43-66.

Maia CJB, Socorro S, Schmitt and Santos CR. (2008): STEAP1 is over- expressed in breast cancer and downregulatedby17beta- estradiolin MCF-7 cells and in the rat mammary gland. Endocrine; 34: 108-16.

Moreaux J, Kassambara A, Hose D and KleinB (2012): STEAP1 is overexpressed in cancers: a promising therapeutic target. Biochem Biophys Res Commun. 14; 429(3-4):148-55. 
Varma M and Jasani B. (2005): Diagnostic utility of immunohistochemistry in morphologically difficult prostate cancer: review of current literature. Histopathology; 47:1-16.

Yang D, Holt GE, Velders MP et al., (2001): Murine six transmembrane epithelial antigen of the prostate, prostate stem cell antigen, and prostate-specific membrane antigen: prostate-specific cell-surface antigens highly expressed in prostate cancer of transgenic adenocarcinoma mouse prostate mice. Cancer Res; 61: 5857-60.

\section{How to cite this article:}

Fatma, M.K.H. Ibrahim, Elsayed A. Ali, Maha M.F. Shamloula, Mohamed M. Shareef and Asmaa E. Bedeer. 2018. Six Transmembrane Epithelial Antigen of Prostate (STEAP1) Immunohistochemical Expression in Neoplastic and Non-Neoplastic Epithelial Lesion of Prostate. Int.J.Curr.Microbiol.App.Sci. 7(10): 606-618. doi: https://doi.org/10.20546/ijcmas.2018.710.068 